\title{
Enhanced Spatial Mapping of Histone Proteoforms in Human Kid- ney Through MALDI-MSI by High-Field UHMR Orbitrap Detection
}

Kevin J. Zemaitis, ${ }^{1}$ Dušan Veličković, ${ }^{1}$ William Kew, ${ }^{1}$ Kyle L. Fort, ${ }^{2}$ Maria Reinhardt-Szyba, ${ }^{2}$ Annapurna Pamreddy, ${ }^{3}$ Yanli Ding, ${ }^{4}$ Dharam Kaushik, ${ }^{5}$ Kumar Sharma, ${ }^{3,6}$ Alexander A. Makarov, ${ }^{2,7}$ Mowei Zhou, ${ }^{1}$ and Ljiljana Paša-Tolić ${ }^{1}$

[1] Environmental Molecular Sciences Laboratory, Pacific Northwest National Laboratory, Richland, Washington 99352, United States

[2] Thermo Fisher Scientific (Bremen) GmbH, 28199 Bremen, Germany

[3] Center for Renal Precision Medicine, Department of Medicine, University of Texas Health, San Antonio, Texas 78284, United States

[4] Department of Pathology and Laboratory Medicine, University of Texas Health, San Antonio, Texas 78284, United States

[5] Department of Urology, University of Texas Health, San Antonio, Texas 78284, United States

[6] Audie L. Murphy Memorial VA Hospital, South Texas Veterans Health Care System, San Antonio, Texas 78284, United States

[7] Biomolecular Mass Spectrometry and Proteomics, Bijvoet Center for Biomolecular Research and Utrecht Institute for Pharmaceutical Sciences, University of Utrecht, Utrecht 3584, The Netherlands

ABSTRACT: Core histones including H2A, H2B, H3, and $\mathrm{H} 4$ are key modulators of cellular repair, transcription, and replication within eukaryotic cells, playing vital roles within the pathogenesis of disease and cellular responses to environmental stimuli. Traditional mass spectrometry (MS) based bottom-up and top-down proteomics allows for the comprehensive identification of proteins and of post-translational modification (PTM) harboring proteoforms. However, these methodologies have difficulties preserving near cellular spatial distributions because they typically require laser capture microdissection (LCM) and advanced sample preparation techniques. Herein, we coupled a matrix-assisted laser desorption/ionization (MALDI) source with a Thermo Scientific Q Exactive HF Orbitrap MS upgraded with ultra-high mass range (UHMR) boards for the first demonstration of complementary high-resolution accurate mass (HR/AM) measurements of proteoforms up to $16.5 \mathrm{kDa}$ directly from tissue using this benchtop mass spectrometer. The platform achieved isotopic resolution throughout the detected mass range, providing confident assignments of proteoforms with low ppm mass error and considerable increase in duty cycle over other Fourier transform mass analyzers. Proteoform mapping of core histones was demonstrated on sections of human kidney at near-cellular spatial resolution, with several key distributions of histone and other proteoforms noted within both healthy biopsy and a section from a renal cell carcinoma (RCC) containing nephrectomy. The use of MALDI-MS imaging (MSI) for proteoform mapping demonstrates several steps towards high-throughput accurate identification of proteoforms and provides a new tool for mapping biomolecule distributions throughout tissue sections in extended mass ranges.

Within eukaryotic cells, regulation of DNA has key modulatory mechanisms which promote repair, transcription, and replication. Conformational changes within the conserved structure of chromatin stems from the nucleosome, which is comprised of four highly dynamic core histones (H2A, H2B, H3, and H4). ${ }^{1,2}$ The backbone of these core histones, and N-terminal tail, which directly interacts with DNA, have been found to harbor multiple post-translational modifications (PTMs) resulting in a multitude of unique proteoforms. ${ }^{3,4}$ Each proteoform potentially serves unique biological function based upon the nucleosomes conformational change via histone PTMs, ultimately resulting in a highly complex histone code, ${ }^{5}$ and epigenetic interactions ${ }^{6,7}$ These chromatin-related proteins are prime examples of dynamic modulators of pathogenesis and cellular repair within eukaryotic lifeforms, and have been extensively studied through traditional proteomic methodologies. ${ }^{8}$

While functional studies of histones have been accomplished at the peptide level using bottom-up (BU) or middle-down (MD) mass spectrometry (MS), proteoform level identification solely from peptide level datasets remains a highly challenging aspect reliant on correlation based bioinformatics tools. ${ }^{9}$ The vast majority of proteoform level characterizations are completed by top-down (TD) analyses of intact proteins. ${ }^{10,11}$ Recent advances combining laser capture microdissection (LCM) with TD-MS enabled identification of greater than 400 proteoforms from roughly 250 cells. ${ }^{12}$, 13 However, such analyses require isolation of cells or tissue sections for protein extraction and subsequent liquid chromatography mass spectrometry (LC/MS), which have high specificity but rela-

tively low throughput This limits the feasibility and resolution of whole tissue LCM based LC/MS by microPOTS ${ }^{14}$ or nanoPOTS ${ }^{15}$, and with the loss of cellular context, challenges arise for interrogation of the native heterogeneity in a spatially resolved manner. To 
this end, mass spectrometry imaging (MSI) has emerged as a powerful high-throughput supplement capable of non-targeted, spatially resolved measurements of biomolecules directly from tissue. ${ }^{16}$

Various modalities have the capability of creating complementary ion images localizing not only xenobiotics, metabolites, lipids, and peptides - but also intact proteoforms. ${ }^{17}$ Despite the widespread proteomic applications within MSI, the path forward is a challenging multidisciplinary effort reliant on instrument and method development. ${ }^{18}$ Several ambient imaging approaches have recently gained more traction for protein applications, allowing for the detection and TD dissociation of multiply charged proteoforms and even native protein complexes. ${ }^{19-23}$ Despite these advances, matrix-assisted laser desorption/ ionization (MALDI) remains the most commonly used tool for MSI based proteomic applications. ${ }^{17,24}$ MALDI has been capable of cellular resolution MSI for intact protein analyses for nearly a decade, ${ }^{25,26}$ with several reports documenting distributions of proteins with masses over 100 $\mathrm{kDa}$ to recent upper bounds of $200 \mathrm{kDa}{ }^{27,28}$ However, several distinct challenges arise, both from the sample preparation requirements, and limited proteome coverage afforded by MSI. ${ }^{29}$

MSI of intact proteoforms has a unique set of prerequisites for ionization and instrumentation. However, a trade-off exists within MSI where throughput, spatial resolution, and mass resolution are interrelated variables and often one or more of these variables is compromised.$^{30}$ Histone proteoforms have been directly analyzed from samples of mouse brain via MALDI-MSI. ${ }^{31}$ Several reports have confidently annotated proteoforms directly from tissue using high sensitivity and mass resolution MS. For example, Fourier transform ion cyclotron resonance (FTICR) MS has been utilized for direct proteoform annotation by high resolution accurate mass (HR/AM) alone. However, exhaustive standalone FTICR MSI studies are usually not completed due to time constraints for imaging, despite exhibiting performance not attainable using faster and more commonly implemented linear time of flight (TOF) mass analyzers. ${ }^{32,33}$

Herein, we demonstrate the addition of a novel sample pretreatment through direct acidification of the tissue for the enhanced MALDI-MSI of core histones (H2A, H2B, H3, and H4), all of which can be detected through the first coupling of MALDI to an ultra-high mass range (UHMR) Thermo Scientific ${ }^{\mathrm{TM}} \mathrm{Q}$ Exactive $^{\mathrm{TM}}$ high-field (HF) Orbitrap ${ }^{\mathrm{TM}}$ MS. While several reports have coupled various MALDI sources to Orbitrap MS, this is the first HR/AM demonstration of isotopically resolved proteoforms with high mass accuracy (i.e., low ppm mass error). This platform also demonstrates the same high-resolution performance as FTICR MS, but with significant reduction in requisite detection time per pixel in extended mass ranges. Herein, two distinct samples of human kidney were studied at near-cellular resolution to benchmark the new MSI platform. Key advantages of this instrument are exhibited for proteoform mapping directly from human kidney tissue, utilizing optimized protocols for histone proteoforms. Several differential and characteristic distributions of histone proteoforms within the sections were observed, alongside small protein signatures, which can be utilized as indicators of underlying processes and/or can serve as potential biomarkers of disease and trauma.

\section{EXPERIMENTAL}

Materials and Chemicals:

HPLC grade acetonitrile, isopropanol, and water were purchased from Fisher Scientific (Fairlawn, NJ). Glacial acetic acid (AA, 99.99\%) and trifluoroacetic acid (TFA, 99.5\%) were Optima LC/MS Grade from Fisher Chemical (Fair Lawn, NJ). 200 proof ethanol was acquired from Decon Laboratories (King of Prussia, PA). Chloroform, 2',5'-Dihydroxyacetophenone (2,5-DHA), and cesium iodide was from Sigma Aldrich (St. Louis, MO).

\section{Samples:}

Sectioning was performed at the University of Texas Health Science Center (UTHSC) at San Antonio. The UTHSC institutional review board has approved all the experimental procedures (IRB No: HSC2017035H). Written consent was attained from all patients. Tissue specimens were obtained by surgical resection in the Department of Pathology, UTHSC. Tissue blocks were fresh frozen in liquid nitrogen (without PBS pre-wetting) and stored at $-80{ }^{\circ} \mathrm{C}$ until analysis. The tissue blocks were not embedded and were cryosectioned at $10 \mu \mathrm{m}$ onto indium tin-oxide (ITO) coated slides. Tissue blocks were stored for 3 months for the cancerous segment, prior to sectioning. After cryosectioning tissue segments were shipped on dry ice, and immediately placed within a $-80{ }^{\circ} \mathrm{C}$ freezer. Prior to analysis the cancerous tissue section was stored at $-80{ }^{\circ} \mathrm{C}$ for 2 months.

\section{Lipid Depletion Washes:}

Pretreatment steps were evaluated for optimizing global histone proteoform signal on human kidney and the optimized procedure was as follows. Samples were desiccated under $18 \mathrm{inHg}$ of vacuum for 30 minutes and then washed in fresh solutions of $70 \%$ ethanol for 30 seconds, $100 \%$ ethanol for 30 seconds, Carnoy's solution (6:3:1 v/v ethanol/chloroform/glacial acetic acid) for 2 minutes, $100 \%$ ethanol for 30 seconds, water with $0.2 \%$ TFA for 15 seconds, and immediately washed in $100 \%$ ethanol for 30 seconds. Samples were then dried by stream of nitrogen gas prior to tissue acidification.

Tissue Acidification Pretreatment:

After washing, each slide was directly acidified with a solution of 5\% acetic acid $(v / v)$ in $50 \%$ ethanol using an HTX Technologies M5 Sprayer (Chapel Hill, NC) with a flow rate of $150 \mu \mathrm{L} / \mathrm{min}$, a nozzle temperature of $30.0^{\circ} \mathrm{C}$, and velocity set to $1250 \mathrm{~mm} / \mathrm{min}$ with 10 PSI of nitrogen gas. A 5 second drying period was added between each of the passes. No delocalization was found to occur due to this pretreatment step.

\section{MALDI Matrix Deposition:}

Directly following the pre-extraction of proteins from tissue by acidification, the same sprayer was used to deposit $15 \mathrm{mg} / \mathrm{mL} 2,5$ DHA in $90 \%$ acetonitrile with $0.2 \%$ TFA. The supernatant of the matrix was collected after sonication and centrifugation. The flow rate of matrix was $150 \mathrm{uL} / \mathrm{min}$ with a nozzle temperature of $30.0^{\circ} \mathrm{C}$, velocity was set to $1300 \mathrm{~mm} / \mathrm{min}$ with 10 PSI of nitrogen gas. 
The matrix coverage was calculated to be $277 \mu \mathrm{g} / \mathrm{cm}^{2}$. Matrix was then recrystallized with $5 \%$ acetic acid solution in water at 38.5 ${ }^{\circ} \mathrm{C}$ and dried for 3.5 minutes, using an apparatus similar to that previously reported, ${ }^{34}$ and then immediately analyzed.

Instrumentation:

An elevated pressure (EP) MALDI source with an atmospheric pressure ionization (API) inlet (Spectroglyph LLC, Kennewick, WA) was mounted on a Q Exactive HF Orbitrap MS upgraded with Q Exactive UHMR boards and operated under custom privileges licenses. ${ }^{35,36}$ This UHMR Q Exactive HF MS with the MALDI source mounted is depicted in Supplemental Figure S1, and a more detailed operation and parameters list is included in supplementary information. Briefly, spectra were acquired over the $m / z$ range of 3,500 to 20,000 and these analyses were performed with a custom noise thresholding setting of 1.5 (vs. default 4.54 ). Acquisitions were carried out at a preset resolution of $240 \mathrm{k}$ at $\mathrm{m} / \mathrm{z} 200$ (corresponding to a $512 \mathrm{~ms}$ transient) with 250 laser shots per pixel. External calibration was performed using direct infusion electrospray of $2 \mathrm{mg} / \mathrm{mL}$ solution of cesium iodide in isopropanol/water $(50 \% \mathrm{v} / \mathrm{v})$.

\section{MALDI Data Processing:}

The reduced profile .RAW files were viewed with FreeStyle software (version 1.4) and imported with positional data (.xml) from the Spectroglyph, LLC source to form ion images in SCiLS Lab software by Bruker Daltonics (version 2022a). Each dataset resulted in more than 40,000 pixels. Images were processed after root-mean square normalization within SCiLS Lab. High resolution bright field imaging was completed before MALDI-MSI and after MALDI matrix was washed off and periodic acid-Schiff (PAS) staining was completed using a standard protocol. These images were used to guide pathology of the segments, and a manual segmentation of regions of interest, which was completed within SCiLS Lab. Mean intensities were extracted and exported, then normalized to the relative surface area $\left(\mathrm{mm}^{2}\right)$ of each region for comparison of proteoform levels across tissue. Proteoform error was reported from single isotopologue. Simulations of theoretical isotopic distributions at various transient lengths for several MALDI capable instruments were completed within Peak-by-Peak software by Spectroswiss (Base Edition, version 2021.8.1.b4). ${ }^{37}$

\section{RESULTS AND DISCUSSION}

Benchmarking Proteoform Mapping within Human Kidney by UHMR Q-Exactive HF Orbitrap MS:

While no further sample pretreatment is required after lipid depletion washes to detect core histone proteoforms, additional washes can greatly influence the adducts and detection of protein fingerprints in different tissue types and organisms. ${ }^{31,32,38,39}$ Traditionally, optimization includes varied timing or additional polar aprotic solvent washes (i.e., Carnoy's solution, chloroform, etc.) for removing higher concentrations of lipids and small molecule interferents as well as various degrees of aqueous washes, which can be acidic, to improve desalting. ${ }^{39-43}$ Evaluation and slight modification of existing protocols resulted in a multi-step lipid depletion protocol optimized for the detection of core histones within human kidney, as described in experimental section. Since histones are also highly soluble in acidic aqueous solutions, as noted by several bulk preparation strategies, ${ }^{44}$ an additional step of tissue acidification for pre-extraction prior to matrix deposition was applied to boost sensitivity of these analyses. Often tissue blocks and sections undergo long term storage, and this has been observed to degrade signal intensity. However, after implementation of tissue acidification we were still able to complete a sensitive analysis. Tissue acidification, completed by directly spraying $5 \%$ acetic acid $(v / v)$ prior to matrix deposition, yielded a two-fold enhancement in the total histone signal, as shown for histone H2A in Supplementary Figure S2.

The entire protocol, including lipid depletion and histone enhancement through tissue acidification, was then applied to map histone proteoforms across several sections of human kidney. The resultant average mass spectrum from this imaging analysis is shown in Figure 1, highlighting the observation of several dozen overlapping isotopic distributions of core histone proteoforms with baseline resolution. Increasing complexity is observed ranging from histone $\mathrm{H} 4$ to histone $\mathrm{H} 3$ proteoforms as expected, with histone $\mathrm{H} 3$ having several more methylation (Me) and acetylation (Ac) sites. This range in complexity together with varied transmission efficiency is a putative explanation for varied intensities of histone families detected via MALDI-MSI.

Currently, MALDI-MSI with UHMR Q Exactive HF Orbitrap detection allows for the acquisition of protein signals up to roughly $m / z, 16,500$ directly from kidney tissue. While data was acquired with a mass resolution setting of $240 \mathrm{k}$ (at $\mathrm{m} / \mathrm{z} 200$ ), the $512 \mathrm{~ms}$ transient resulted in an observed experimental resolution of $35 \mathrm{k}$ at $\mathrm{m} / \mathrm{z}, 11,306$, which is in good agreement with theoretically calculated mass resolution. Full magnitude mode $(\mathrm{mFT})$ and absorption mode processing (aFT) of simulations within Peak-by-Peak are shown in Supplementary Figure S3, and Supplementary Table 1. Experimentally observed resolution was found to be consistent with the expected improvement for enhanced FT (eFT) within the entire mass range. ${ }^{45}$ Lowered threshold for noise rejection in this work resulted in a noticeable baseline in Figure 1, while beat pattern and signal decay of protein ions over the duration of the transient lead to imperfect balancing of absorption peak shape in eFT, ${ }^{43}$ revealed in valleys between isotopes sagging below the baseline. These analyses provided baseline resolution of all detected proteoforms, while maintaining a low ppm mass accuracy with external calibration.

The data obtained from this benchmarking is also highly complementary to reports of high-resolution protein MALDI-MSI by $15 \mathrm{~T}$ FTICR, which has been demonstrated to provide mass resolution up to $70 \mathrm{k}$ at $\mathrm{m} / \mathrm{z} 11,000$, requiring a $6.71 \mathrm{~s}$ transient. ${ }^{33}$ Further simulations of isotopic distributions were completed within Peak-by-Peak for 15T FTICR as shown in Supplementary Figure S4, and Supplementary Table 1. To achieve resolution comparable to the UHMR Q Exactive HF performance demonstrated for the acetylated and dimethylated histone $\mathrm{H} 4$, a $3.4 \mathrm{~s}$ transient per pixel ( $0.3 \mathrm{~Hz}$ acquisition) is required for the 15T FTICR-MS with standard mFT processing. Specifically, UHMR Q Exactive HF performance within a $512 \mathrm{~ms}$ transient (2 Hz acquisition) signifies a 6.6-fold improvement in duty cycle for proteoform MALDI-MSI. Future studies within this platform could either utilize more sensitive and less vacuum stable matrixes, profile larger regions of interest, further spatial resolution to cellular and sub-cellular levels, or increase the mass resolution with no significant losses in throughput. 
Similarly, advanced aFT signal processing and/or frequency multiple detection within FTICR MS would significantly boost throughput. ${ }^{46,47}$ However, further examination of experimental and simulated spectra demonstrates the well-known slower reduction in resolution at increasing $\mathrm{m} / \mathrm{z}$ with Orbitrap MS compared to FTICR MS. More specifically, Orbitrap resolving power diminishes proportional to $1 / \sqrt{ } \mathrm{m} / z$, whereas in FTICR resolving power is proportional to $1 / \mathrm{m} / \mathrm{z}^{48}$ This has also been observed previously within single ion experiments of ultra large protein complexes. ${ }^{49}$ Furthermore, with an extended transient of 1.024 seconds (Supplementary Figure S5), the UHMR Q Exactive HF has an observable mass resolution of 70k at $\mathrm{m} / z, 11,306$, allowing for preservation of high mass resolution and near cellular resolution for entire sections of tissue and further demonstrating the exquisite utility of this platform. ${ }^{50}$

High Throughput MALDI-MSI for Near Single Cell Resolution of Whole Samples

The human kidney is an incredibly complex organ with several distinct cell types and functional tissue units, and hundreds of different diseases have various pathophysiological effects throughout the cortex and the medulla. Hence, several methodologies are typically used concurrently with both high spatial and high mass resolution imaging for confident, clinically relevant analyses. ${ }^{51}$ Pathogenesis of disease and trauma have broad ranging consequences spanning hypertension, metabolic stress and injury, as well as endothelial apoptosis. This occurs within the glomeruli, distal and proximal tubules, and crosses the interfacial region into the medullary regions systemically effecting the entire renal system. ${ }^{52,53}$ We have applied MALDI-MSI at near single cellular resolution to account for this complexity and facilitate proteoform informed mapping of the functional tissue units. The profiling of the intrinsic heterogeneity within distinct kidney regions and tissue units allows for the extraction of clinically relevant information.

Due to the high mass resolution being maintained with shorter transients, $20 \mu \mathrm{m}$ spatial resolution was feasible with relatively high throughput. The resultant 39,078 pixels across the tissue section required a calculated $5.56 \mathrm{hrs}$ to complete (accounting for the detection period only), in comparison to an estimated $36.9 \mathrm{hrs}$ that would have been required using mFT processing of $15 \mathrm{~T}$ FTICR transients for comparable resolution at $\mathrm{m} / \mathrm{z}$ 11,306. In a relatively high-throughput manner, unique histone proteoform distributions were observed within tubules in the cortical regions, which can be noted within the expanded ion images in Figure 2. This figure highlights the putatively annotated histone H4 N-Ac K20me2 and H4 N-Ac / Ac proteoforms. Dimethylation at K20 has been previously linked to double stranded DNA damage response. Similarly, several readers of K20me2 are known to recruit various repair proteins with links to cell cycle regulation, which aligns with chronic damage. ${ }^{54-56}$

The PAS staining of this fresh frozen nephrectomy section confirmed pre-existing chronic kidney damage including tubular atrophy and accompanying interstitial fibrosis, indicated through thickened tubular basement membranes and simplified tubular epithelium due to tubular injury resultant in stenosis and other afflictions. ${ }^{57}$ Histological staining also allowed for identification of glomeruli, which were observed to have lower signal intensities for all histone proteoforms relative to the tubular regions. Histone proteoforms, at large, were observed to be colocalized within this analysis, with no distinct observed localizations apparent until a more in-depth profiling was completed. After extraction of proteoform intensities through manual segmentation, significant differential abundances of proteoforms were noted. These results are presented in Figure 3 for H4 N-Ac K20me2 and H4 N-Ac / Ac for the averaged normalized mean intensity for several exemplary tubular regions.

Utilizing this strategy, a general trend was observed that signified certain tubules having differential abundances of histone proteoforms. After normalization of the mean intensities to the area of the extracted regions of interest, the average was taken for several enhanced atrophic regions $(\mathrm{ET}, \mathrm{n}=4)$ and several depleted atrophic tubular regions $(\mathrm{T}, \mathrm{n}=4)$. H4 N-Ac K20me2 proteoform showed increased abundance within a subset of ET regions, as shown within Figure 3. Differential abundance within the average of these various regions of atrophy is a unique observation for this damage marking proteoform. In ET regions proteoform intensities of putative $\mathrm{H} 4 \mathrm{~N}-\mathrm{Ac}$ K20me 2 were found to be significantly greater (p-value of 0.0056; two-sample t-test, one tail). Diacetylated histone H4 displayed similar differential abundances (p-value of 0.023 ; two-sample t-test, one tail). These results can provide putative links to varying degrees of tubular atrophy. Further study is warranted for increased statistical power and to further differentiate tubular regions (i.e., distal and proximal tubules) within the cortex.

While histone $\mathrm{H} 4 \mathrm{~N}-\mathrm{ac} \mathrm{K} 20 \mathrm{me} 2$ has been noted in several reports as the most abundant $\mathrm{H} 4$ proteoform, ${ }^{32,39,58}$ sole annotation by HR/AM measurements are tentative. This lends credence to multiplexed LCM based proteomics and alternative TD-MS strategies such as implementation of MALDI in-source decay (ISD) or ultraviolet photodissociation (UVPD) both of which have been demonstrated within lower mass ranges on MALDI enabled Orbitrap MS. ${ }^{59}$ Routine TD fragmentation of proteoforms has not yet been demonstrated directly from tissue for MALDI analyses, but dissociation methods are more commonly for ambient ionization techniques which create multiply charged protein ions. ${ }^{21,23,60}$ Nonetheless, the variance noted within the proteoforms detected by MALDI-MSI highlights the stability and promise of this platform for the mapping of distinct (histone) proteoform signatures, as exemplified above within regions of tubular atrophy.

Case Study: MALDI-MSI on a Section of Kidney from a Renal Cell Carcinoma Nephrectomy:

Hereditary disorders, as well as external risk factors such as smoking and exposure to toxins, play roles in the pathogenesis of cancer, suggesting a strong link to the epigenetics and histone profiles. ${ }^{4}$ Several studies noted the importance of epi-proteomic profiling of clinical samples and implicated histone proteoforms as potential biomarkers of disease utilizing techniques such as MALDIFTICR and MALDI-TOF imaging for clinical research. ${ }^{32,58}$ Renal cell carcinoma (RCC) is the most common type of kidney cancer in adults, and typically results in a nephrectomy. As the disease progresses from within the linings of the tubules, discrete tumors are often formed. ${ }^{61}$ While the RCC tissue section presented herein was stored for an extended period ( 2 months), histone proteoforms were directly detected from the tissue through the optimized MALDI-MSI protocol.

Interestingly, bulk histone distributions within the healthy region were found to be consistent with several other profiled kidney sections. However, distinct areas of increased abundance of core histone proteoforms were found within the RCC tumor segment, which exists within confined borders within this section (outlined in Figure 4A and 4B). Distinct enhancements of several 
proteoforms are noted within this border region of the RCC segment at the interface of the healthy tissue, as shown within Supplementary Figure S6. Note that if bulk proteomics measurements were made, or even spatially resolved LCM based LC/MS analyses, without a priori knowledge of the sample, these enhanced abundances at the edge of the tumor would have been missed. This finding highlights the importance of spatially resolved proteomic measurements in clinical and translational research.

Ion images from distributions of putative $\mathrm{H} 4 \mathrm{~N}-\mathrm{Ac} \mathrm{K} 20 \mathrm{me} 2, \mathrm{H} 4 \mathrm{~N}-\mathrm{Ac} / \mathrm{Ac}$, and H4 N-Ac / Ac K20me2 proteoforms are highlighted in Figures 4C, 4D, and 4E, respectively. These results warrant further studies for the determination of the epigenetic relevance to RCC progression, as normal levels of the histone proteoforms within the healthy region are significantly lower when compared to the RCC region. As before, the base peak of the analysis corresponds to an annotated histone $\mathrm{H} 4 \mathrm{~N}$-ac $\mathrm{K} 20 \mathrm{me} 2$ proteoform (Fig. 4C), but interestingly all detected H4 histone proteoforms show unique distributions within gradients in the RCC tissue segment.

An early MALDI-TOF MSI analysis with tandem immunohistostaining showed high levels of enhancement of K20me2 through microvascular invasion after hepatocellular carcinoma (HCC) removal. This PTM was identified as a putative biomarker of postoperative recurrence of $\mathrm{HCCs} .{ }^{58} \mathrm{As} \mathrm{K} 20 \mathrm{me} 2$ is an indicator of damage and trauma, the enhancement within the border region of the RCC segments is also logical, and could be utilized within additional studies as a potential biomarker once validated through supplementary methods and clinically relevant sample sizes. Additionally, colocalizations of 40S ribosomal protein S30 at $m / z$ 6647.9 has broad links to cell plasticity. ${ }^{62,63}$ This annotated protein was enhanced within the healthy cortical region containing a subset of tubules and showed increased intensity within the RCC segment. This demonstration of proteoform informed MALDI-MSI by UHMR Q Exactive HF further expands upon previous MALDI-TOF imaging completed on clear cell RCC, which demonstrated the complexities of annotation of proteins without sufficient resolution for isotopic resolution. ${ }^{64}$ This illustrates the utility of MALDIMSI for broad profiling of proteoforms within entire tissue sections enabled by high resolution FTMS.

\section{CONCLUSIONS}

We present the first implementation of MALDI-MSI on an UHMR-Orbitrap MS resulting in the detection of proteoforms of up to $16.5 \mathrm{kDa}$ directly from human kidney tissue sections at near-cellular resolution. Through the optimization of lipid depletion washes, and the addition of a novel tissue pre-extraction step, a sensitive spatial analysis of a range of core histone proteoforms was achieved on fresh frozen tissue, which has been in storage for various periods. A UHMR Q Exactive HF also provided sufficient mass resolution for the isotopically resolved measurements at $2 \mathrm{~Hz}$ sampling rate, comparable at all levels to $15 \mathrm{~T}$ FTICR for MALDI-MSI of proteoforms within an extended mass range. In fact, this new platform outperforms FTICR with a reported 6.6-fold increase in duty cycle per pixel for equivalent observed resolution in normal modes of operation. These gains within extended mass proteoform mapping by MALDI align well within goals of clinical and translational studies which require large cohorts for biological relevance.

This platform also permitted the annotation of proteoforms based upon HR/AM measurements matched against theoretically calculated isotopic distributions. The UHMR Q Exactive HF exhibited exceptional stability with mass error below 3 ppm within the calibrated mass range using external calibration. Since on-tissue tandem MS of low-charge state ions is challenging by existing methods, subsequent TD-MS experiments are still required to confidently identify various proteoforms. To address this challenge and facilitate proteoform-aware mapping of tissues with high-throughput and near-cellular resolution, we are currently integrating MALDI-MSI with LC/MS micro-proteomics (i.e., microPOTS or nanoPOTS) and concurrently we are developing advanced dissociation techniques such as UVPD for proteoforms $(>m / z$ 6000) desorbed and ionized by MALDI. Overall, the utility of MALDI on an UHMR Q Exactive HF is well positioned to provide ultra-high mass resolution within extended ranges at 1-2 Hz sampling rate for significant throughput enhancement. This level of performance will also enable future developmental pushes to expand the detectable mass range to larger proteins and proteoforms. Future studies with large cohorts will also be feasible within several days (versus several weeks currently), making proteoform informed FTMS-MSI more competitive to faster TOF-MSI and/or enable future expansion and development of cellular and sub-cellular imaging modalities.

\section{ASSOCIATED CONTENT}

Supporting Information

Supplementary Information: (.docx)

\section{AUTHOR INFORMATION}

Corresponding Author

* Ljiljana Paša-Tolić - Environmental Molecular Sciences Laboratory, Pacific Northwest National Laboratory, Richland, Washington 99354, United States

Email: ljiljana.pasatolic@pnnl.gov

\section{Author Contributions}

L.P.T., M.Z., D.V., W.K., and K.J.Z. conceptualized the research. K.J.Z., M.Z., and W.K. through collaboration with K.L.F, M.R.S., and A.A.M. converted the Q-Exactive HF to have UHMR capability, and K.J.Z., D.V., M.Z., and W.K. validated and developed the mass spectrometry methods. K.J.Z. performed the sample preparation and MALDI-MSI experiments. A.P., Y.D., D.K., and K.S. provided the samples within this research. K.J.Z. and D.V. performed the data analysis, and K.J.Z. performed the formal analysis and visualization with 
input from Y.D., K.S. as well as D.V., M.Z., W.K., and L.P.T. The original manuscript was drafted by K.J.Z., and K.J.Z., D.V., M.Z., W.K., and L.P.T reviewed and edited with input from K.L.F, M.R.S., A.A.M. and A.P., Y.D., D.K., K.S. as well. L.P.T. and W.K. acquired funds for research. All authors have given approval to the final version of the manuscript.

Notes

K.L.F, M.R.S., and A.A.M. declare the following competing financial interest(s): these authors are employees of Thermo Fisher Scientific, manufacturer of Orbitrap mass spectrometers.

\section{ACKNOWLEDGMENT}

The authors would also like to acknowledge Dr. Marija Veličković for assistance within the histological staining and microscopy, and Dr. Mikhail Belov at Spectroglyph, LLC, as well as Dr. Gordon Anderson and Chris Anderson at GAA Custom Electronics, LLC for technical support for the MALDI source. The authors would also like to thank Drs. Yury Tsybin, Konstantin Nagornov, and Anton Kozhinov at Spectroswiss for support within the Peak-By-Peak software. This work was performed at the Environmental Molecular Science Laboratory (EMSL), a Department of Energy (DOE) Office of Science User Facility sponsored by the Office of Biological and Environmental Research. This research was funded by the National Institutes of Health (NIH) Common Fund, Human Biomolecular Atlas Program (HuBMAP) grant UG3CA256959-01, doi.org/10.46936/staf.proj.2020.51770/60000309 (L.P.T.) and by the intramural program on project award doi.org/10.46936/intm.proj.2019.51159/60000152 (W.K) at EMSL (grid.436923.9) operated under Contract No. DE-AC05-76RL01830.

\section{REFERENCES}

1. Strahl, B. D.; Allis, C. D., The language of covalent histone modifications. Nature 2000, 403 (6765), 41-45.

2. Gillette, T. G.; Hill, J. A., Readers, writers, and erasers: chromatin as the whiteboard of heart disease. Circ Res 2015, 116 (7), 1245-1253.

3. $\quad$ Lusser, A.; Kölle, D.; Loidl, P., Histone acetylation: lessons from the plant kingdom. Trends in Plant Science 2001, 6 (2), $59-65$.

4. Zhao, Z.; Shilatifard, A., Epigenetic modifications of histones in cancer. Genome Biology 2019, 20 (1), 245.

5. Jenuwein, T.; Allis, C. D., Translating the Histone Code. Science 2001, 293 (5532), 1074-1080.

6. Smith, L. M.; Kelleher, N. L.; Linial, M.; Goodlett, D.; Langridge-Smith, P.; Ah Goo, Y.; Safford, G.; Bonilla*, L.; Kruppa, G.; Zubarev, R.; Rontree, J.; Chamot-Rooke, J.; Garavelli, J.; Heck, A.; Loo, J.; Penque, D.; Hornshaw, M.; Hendrickson, C.; PasaTolic, L.; Borchers, C.; Chan, D.; Young*, N.; Agar, J.; Masselon, C.; Gross*, M.; McLafferty, F.; Tsybin, Y.; Ge, Y.; Sanders*, I.; Langridge, J.; Whitelegge*, J.; Marshall, A.; The Consortium for Top Down, P., Proteoform: a single term describing protein complexity. Nature Methods 2013, 10 (3), 186-187.

7. Smith, L. M.; Kelleher, N. L., Proteoforms as the next proteomics currency. Science 2018, 359 (6380), 1106.

8. Lu, C.; Coradin, M.; Porter, E. G.; Garcia, B. A., Accelerating the Field of Epigenetic Histone Modification Through Mass Spectrometry\&\#x2013;Based Approaches. Molecular \& Cellular Proteomics 2021, 20.

9. $\quad$ Bludau, I.; Frank, M.; Dörig, C.; Cai, Y.; Heusel, M.; Rosenberger, G.; Picotti, P.; Collins, B. C.; Röst, H.; Aebersold, R., Systematic detection of functional proteoform groups from bottom-up proteomic datasets. Nature Communications 2021, 12 (1), 3810.

10. Toby, T. K.; Fornelli, L.; Kelleher, N. L., Progress in Top-Down Proteomics and the Analysis of Proteoforms. Annual Review of Analytical Chemistry 2016, 9 (1), 499-519.

11. Chen, B.; Brown, K. A.; Lin, Z.; Ge, Y., Top-Down Proteomics: Ready for Prime Time? Analytical Chemistry 2018, 90 (1), 110-127.

12. Zhou, M.; Uwugiaren, N.; Williams, S. M.; Moore, R. J.; Zhao, R.; Goodlett, D.; Dapic, I.; Paša-Tolić, L.; Zhu, Y., Sensitive Top-Down Proteomics Analysis of a Low Number of Mammalian Cells Using a Nanodroplet Sample Processing Platform. Anal Chem 2020, 92 (10), 7087-7095.

13. Lubeckyj, R. A.; Sun, L., Laser capture microdissection-capillary zone electrophoresis-tandem mass spectrometry (LCM-CZEMS/MS) for spatially resolved top-down proteomics: a pilot study of zebrafish brain. Molecular Omics 2022.

14. Xu, K.; Liang, Y.; Piehowski, P. D.; Dou, M.; Schwarz, K. C.; Zhao, R.; Sontag, R. L.; Moore, R. J.; Zhu, Y.; Kelly, R. T., Benchtop-compatible sample processing workflow for proteome profiling of $<100$ mammalian cells. Anal Bioanal Chem 2019, 411 (19), 4587-4596.

15. Zhu, Y.; Piehowski, P. D.; Zhao, R.; Chen, J.; Shen, Y.; Moore, R. J.; Shukla, A. K.; Petyuk, V. A.; Campbell-Thompson, M.; Mathews, C. E.; Smith, R. D.; Qian, W.-J.; Kelly, R. T., Nanodroplet processing platform for deep and quantitative proteome profiling of 10-100 mammalian cells. Nature Communications 2018, 9 (1), 882.

16. Buchberger, A. R.; DeLaney, K.; Johnson, J.; Li, L., Mass Spectrometry Imaging: A Review of Emerging Advancements and Future Insights. Analytical chemistry 2018, 90 (1), 240-265.

17. Aichler, M.; Walch, A., MALDI Imaging mass spectrometry: current frontiers and perspectives in pathology research and practice. Laboratory Investigation 2015, 95 (4), 422-431.

18. Aebersold, R.; Mann, M., Mass-spectrometric exploration of proteome structure and function. Nature 2016, 537 (7620), 347-

355.

19. Hsu, C.-C.; Chou, P.-T.; Zare, R. N., Imaging of Proteins in Tissue Samples Using Nanospray Desorption Electrospray Ionization Mass Spectrometry. Analytical Chemistry 2015, 87 (22), 11171-11175.

20. Towers, M. W.; Karancsi, T.; Jones, E. A.; Pringle, S. D.; Claude, E., Optimised Desorption Electrospray Ionisation Mass Spectrometry Imaging (DESI-MSI) for the Analysis of Proteins/Peptides Directly from Tissue Sections on a Travelling Wave Ion Mobility Q-ToF. J Am Soc Mass Spectrom 2018, 29 (12), 2456-2466.

21. Griffiths, R. L.; Konijnenberg, A.; Viner, R.; Cooper, H. J., Direct Mass Spectrometry Analysis of Protein Complexes and Intact Proteins up to $>70 \mathrm{kDa}$ from Tissue. Anal Chem 2019, 91 (11), 6962-6966.

22. Hale, O. J.; Cooper, H. J., Native Mass Spectrometry Imaging of Proteins and Protein Complexes by Nano-DESI. Anal Chem 2021, 93 (10), 4619-4627. 
23. Su, P.; McGee, J. P.; Durbin, K. R.; Hollas, M. A. R.; Yang, M.; Neumann, E. K.; Allen, J. L.; Drown, B. S.; Butun, F. A.; Greer, J. B.; Early, B. P.; Fellers, R. T.; Spraggins, J. M.; Laskin, J.; Camarillo, J. M.; Kafader, J. O.; Kelleher, N. L., Direct Imaging and Identification of Proteoforms up to $70 \mathrm{kDa}$ from Human Tissue. bioRxiv 2021, 2021.12.07.471638.

24. McDonnell, L. A.; Corthals, G. L.; Willems, S. M.; van Remoortere, A.; van Zeijl, R. J. M.; Deelder, A. M., Peptide and protein imaging mass spectrometry in cancer research. Journal of Proteomics 2010, 73 (10), 1921-1944.

25. Zavalin, A.; Todd, E. M.; Rawhouser, P. D.; Yang, J.; Norris, J. L.; Caprioli, R. M., Direct imaging of single cells and tissue at sub-cellular spatial resolution using transmission geometry MALDI MS. Journal of mass spectrometry : JMS 2012, 47 (11), i-i.

26. Zavalin, A.; Yang, J.; Hayden, K.; Vestal, M.; Caprioli, R. M., Tissue protein imaging at $1 \mu \mathrm{m}$ laser spot diameter for high spatial resolution and high imaging speed using transmission geometry MALDI TOF MS. Anal Bioanal Chem 2015, 407 (8), $2337-42$.

27. van Remoortere, A.; van Zeijl, R. J.; van den Oever, N.; Franck, J.; Longuespée, R.; Wisztorski, M.; Salzet, M.; Deelder, A. M.; Fournier, I.; McDonnell, L. A., MALDI imaging and profiling MS of higher mass proteins from tissue. J Am Soc Mass Spectrom 2010, 21 (11), 1922-9.

28. Liu, H.; Han, M.; Li, J.; Qin, L.; Chen, L.; Hao, Q.; Jiang, D.; Chen, D.; Ji, Y.; Han, H.; Long, C.; Zhou, Y.; Feng, J.; Wang, X., A Caffeic Acid Matrix Improves In Situ Detection and Imaging of Proteins with High Molecular Weight Close to 200,000 Da in Tissues by Matrix-Assisted Laser Desorption/Ionization Mass Spectrometry Imaging. Anal Chem 2021.

29. Han, J.; Permentier, H.; Bischoff, R.; Groothuis, G.; Casini, A.; Horvatovich, P., Imaging of protein distribution in tissues using mass spectrometry: An interdisciplinary challenge. TrAC Trends in Analytical Chemistry 2019, 112, 13-28.

30. Spraggins, J. M.; Rizzo, D. G.; Moore, J. L.; Noto, M. J.; Skaar, E. P.; Caprioli, R. M., Next-generation technologies for spatial proteomics: Integrating ultra-high speed MALDI-TOF and high mass resolution MALDI FTICR imaging mass spectrometry for protein analysis. Proteomics 2016, 16 (11-12), 1678-1689.

31. Lahiri, S.; Sun, N.; Solis-Mezarino, V.; Fedisch, A.; Ninkovic, J.; Feuchtinger, A.; Götz, M.; Walch, A.; Imhof, A., In situ detection of histone variants and modifications in mouse brain using imaging mass spectrometry. PROTEOMICS 2016, 16 (3), $437-447$.

32. Dilillo, M.; Ait-Belkacem, R.; Esteve, C.; Pellegrini, D.; Nicolardi, S.; Costa, M.; Vannini, E.; Graaf, E. L. d.; Caleo, M.; McDonnell, L. A., Ultra-High Mass Resolution MALDI Imaging Mass Spectrometry of Proteins and Metabolites in a Mouse Model of Glioblastoma. Sci Rep 2017, 7 (1), 603-603.

33. Prentice, B. M.; Ryan, D. J.; Van de Plas, R.; Caprioli, R. M.; Spraggins, J. M., Enhanced Ion Transmission Efficiency up to m/ z 24000 for MALDI Protein Imaging Mass Spectrometry. Analytical chemistry 2018, 90 (8), 5090-5099.

34. Yang, J.; Caprioli, R. M., Matrix Sublimation/Recrystallization for Imaging Proteins by Mass Spectrometry at High Spatial Resolution. Analytical Chemistry 2011, 83 (14), 5728-5734.

35. Fort, K. L.; van de Waterbeemd, M.; Boll, D.; Reinhardt-Szyba, M.; Belov, M. E.; Sasaki, E.; Zschoche, R.; Hilvert, D.; Makarov, A. A.; Heck, A. J. R., Expanding the structural analysis capabilities on an Orbitrap-based mass spectrometer for large macromolecular complexes. Analyst 2018, 143 (1), 100-105.

36. van de Waterbeemd, M.; Tamara, S.; Fort, K. L.; Damoc, E.; Franc, V.; Bieri, P.; Itten, M.; Makarov, A.; Ban, N.; Heck, A. J. R., Dissecting ribosomal particles throughout the kingdoms of life using advanced hybrid mass spectrometry methods. Nature Communications 2018, 9 (1), 2493.

37. Nagornov, K. O.; Kozhinov, A. N.; Gasilova, N.; Menin, L.; Tsybin, Y. O., Transient-Mediated Simulations of FTMS Isotopic Distributions and Mass Spectra to Guide Experiment Design and Data Analysis. Journal of the American Society for Mass Spectrometry 2020, 31 (9), 1927-1942.

38. $\quad$ Spraggins, J. M.; Rizzo, D. G.; Moore, J. L.; Rose, K. L.; Hammer, N. D.; Skaar, E. P.; Caprioli, R. M., MALDI FTICR IMS of Intact Proteins: Using Mass Accuracy to Link Protein Images with Proteomics Data. J Am Soc Mass Spectrom 2015, 26 (6), 974-85.

39. Piga, I.; Heijs, B.; Nicolardi, S.; Giusti, L.; Marselli, L.; Marchetti, P.; Mazzoni, M. R.; Lucacchini, A.; McDonnell, L. A., Ultra-high resolution MALDI-FTICR-MSI analysis of intact proteins in mouse and human pancreas tissue. International Journal of Mass Spectrometry 2019, 437, 10-16.

40. Lemaire, R.; Wisztorski, M.; Desmons, A.; Tabet, J. C.; Day, R.; Salzet, M.; Fournier, I., MALDI-MS Direct Tissue Analysis of Proteins: Improving Signal Sensitivity Using Organic Treatments. Analytical Chemistry 2006, 78 (20), $7145-7153$.

41. Seeley, E. H.; Oppenheimer, S. R.; Mi, D.; Chaurand, P.; Caprioli, R. M., Enhancement of protein sensitivity for MALDI imaging mass spectrometry after chemical treatment of tissue sections. Journal of the American Society for Mass Spectrometry 2008, 19 (8), 1069-1077.

42. Deutskens, F.; Yang, J.; Caprioli, R. M., High spatial resolution imaging mass spectrometry and classical histology on a single tissue section. Journal of Mass Spectrometry 2011, 46 (6), 568-571.

43. Rešetar Maslov, D.; Svirkova, A.; Allmaier, G.; Marchetti-Deschamann, M.; Kraljević Pavelić, S., Optimization of MALDITOF mass spectrometry imaging for the visualization and comparison of peptide distributions in dry-cured ham muscle fibers. Food Chemistry 2019, 283, 275-286.

44. Shechter, D.; Dormann, H. L.; Allis, C. D.; Hake, S. B., Extraction, purification and analysis of histones. Nature Protocols 2007, 2 (6), 1445-1457.

45. Lange, O.; Damoc, E.; Wieghaus, A.; Makarov, A., Enhanced Fourier transform for Orbitrap mass spectrometry. International Journal of Mass Spectrometry 2014, 369, 16-22.

46. Shaw, J. B.; Gorshkov, M. V.; Wu, Q.; Paša-Tolić, L., High Speed Intact Protein Characterization Using 4X Frequency Multiplication, Ion Trap Harmonization, and 21 Tesla FTICR-MS. Analytical Chemistry 2018, 90 (9), 5557-5562.

47. Park, S.-G.; Anderson, G. A.; Bruce, J. E., Parallel Detection of Fundamental and Sixth Harmonic Signals Using an ICR Cell with Dipole and Sixth Harmonic Detectors. Journal of the American Society for Mass Spectrometry 2020, 31 (3), $719-726$.

48. Zubarev, R. A.; Makarov, A., Orbitrap mass spectrometry. ACS Publications: 2013.

49. McGee, J. P.; Melani, R. D.; Yip, P. F.; Senko, M. W.; Compton, P. D.; Kafader, J. O.; Kelleher, N. L., Isotopic Resolution of Protein Complexes up to 466 kDa Using Individual Ion Mass Spectrometry. Analytical Chemistry 2021, 93 (5), $2723-2727$.

50. Denisov, E.; Damoc, E.; Makarov, A., Exploring frontiers of orbitrap performance for long transients. International Journal of Mass Spectrometry 2021, 466, 116607. 
51. Kruse, A. R. S.; Spraggins, J. M., Uncovering Molecular Heterogeneity in the Kidney With Spatially Targeted Mass Spectrometry. Frontiers in Physiology 2022, 13.

52. Matovinović, M. S., 1. Pathophysiology and Classification of Kidney Diseases. EJIFCC 2009, 20 (1), 2-11.

53. Dumas, S. J.; Meta, E.; Borri, M.; Luo, Y.; Li, X.; Rabelink, T. J.; Carmeliet, P., Phenotypic diversity and metabolic specialization of renal endothelial cells. Nature Reviews Nephrology 2021, 17 (7), 441-464.

54. Paquin, K. L.; Howlett, N. G., Understanding the Histone DNA Repair Code: H4K20me2 Makes Its Mark. Mol Cancer Res 2018, $16(9), 1335-1345$.

55. Bannister, A. J.; Schneider, R.; Kouzarides, T., Histone Methylation: Dynamic or Static? Cell 2002, 109 (7), $801-806$.

56. Wang, T.; Holt, M. V.; Young, N. L., The histone H4 proteoform dynamics in response to SUV4-20 inhibition reveals single molecule mechanisms of inhibitor resistance. Epigenetics \& Chromatin 2018, 11 (1), 29.

57. Lusco, M. A.; Fogo, A. B.; Najafian, B.; Alpers, C. E., AJKD Atlas of Renal Pathology: Tubular Atrophy. American Journal of Kidney Diseases 2016, 67 (6), e33-e34.

58. $\quad$ Poté, N.; Alexandrov, T.; Le Faouder, J.; Laouirem, S.; Léger, T.; Mebarki, M.; Belghiti, J.; Camadro, J.-M.; Bedossa, P.; Paradis, V., Imaging mass spectrometry reveals modified forms of histone $\mathrm{H} 4$ as new biomarkers of microvascular invasion in hepatocellular carcinomas. Hepatology 2013, 58 (3), 983-994.

59. Dilillo, M.; de Graaf, E. L.; Yadav, A.; Belov, M. E.; McDonnell, L. A., Ultraviolet Photodissociation of ESI- and MALDIGenerated Protein Ions on a Q-Exactive Mass Spectrometer. J Proteome Res 2019, 18 (1), 557-564.

60. Garza, K. Y.; Feider, C. L.; Klein, D. R.; Rosenberg, J. A.; Brodbelt, J. S.; Eberlin, L. S., Desorption Electrospray Ionization Mass Spectrometry Imaging of Proteins Directly from Biological Tissue Sections. Anal Chem 2018, 90 (13), $7785-7789$.

61. Petejova, N.; Martinek, A., Renal cell carcinoma: Review of etiology, pathophysiology and risk factors. Biomed Pap Med Fac Univ Palacky Olomouc Czech Repub 2016, 160 (2), 183-94.

62. Ogata, K.; Ohno, R.; Terao, K.; Endo, Y., An ATPase center of rat liver 30S-5SRNP particles. J Biochem 2000, 128 (1), 1-9.

63. Trakman, L.; Hewson, C.; Burdach, J.; Morris, K. V., RNA Directed Modulation of Phenotypic Plasticity in Human Cells.

PLoS One 2016, 11 (4), e0152424.

64. Jones, E. E.; Powers, T. W.; Neely, B. A.; Cazares, L. H.; Troyer, D. A.; Parker, A. S.; Drake, R. R., MALDI imaging mass spectrometry profiling of proteins and lipids in clear cell renal cell carcinoma. PROTEOMICS 2014, 14 (7-8), 924-935.

Authors are required to submit a graphic entry for the Table of Contents (TOC) that, in conjunction with the manuscript title, should give the reader a representative idea of one of the following: A key structure, reaction, equation, concept, or theorem, etc., that is discussed in the manuscript. Consult the journal's Instructions for Authors for TOC graphic specifications.

TOC Graphic

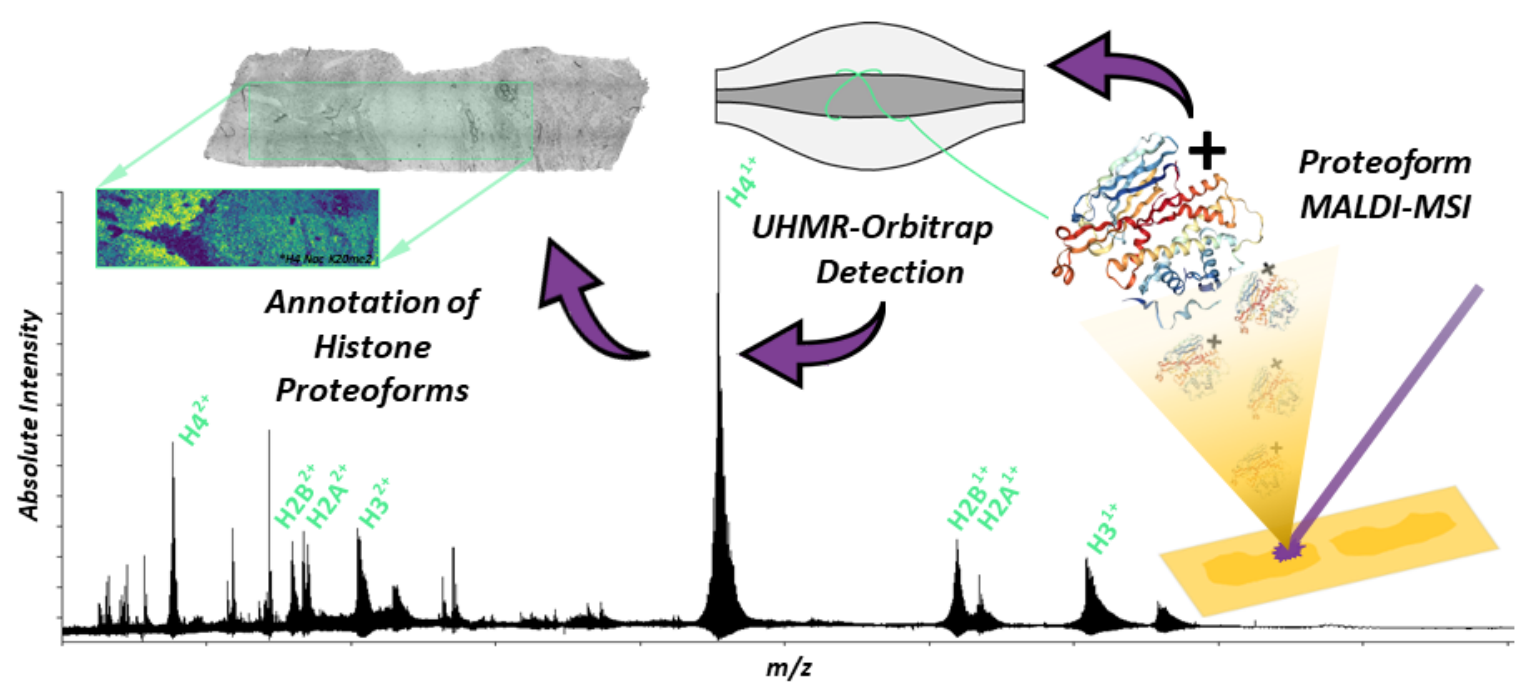




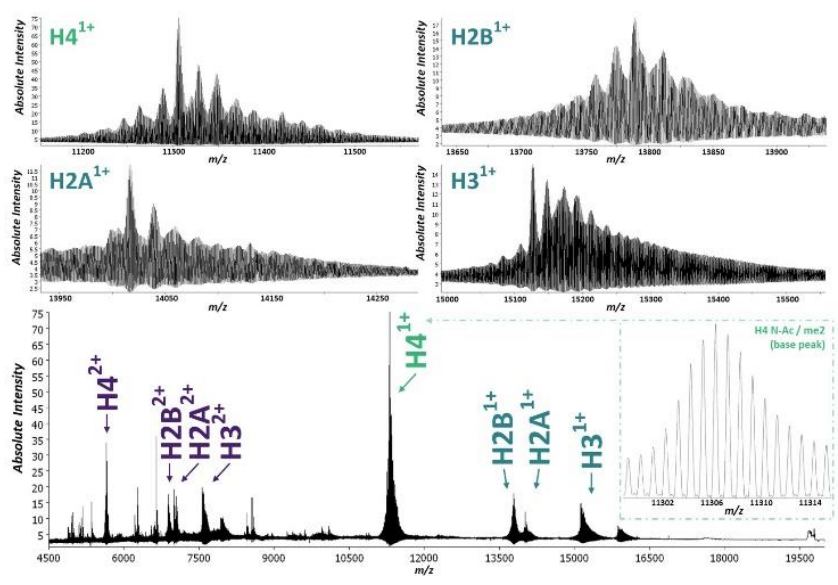

Figure 1. Full mass spectrum from the proteoform mapping of human kidney at $40 \mu \mathrm{m}$ obtained by averaging a total of 86,189 pixels highlighting both, the singly and doubly charged core histone proteoform ion clusters. Expanded views of each singly charged histone (H2A, H2B, H3, H4) proteoform family are also shown with isotopic resolution throughout the entire $\mathrm{m} / z$ range. The only other near isobaric species other than histone proteoforms are located within the histone $\mathrm{H} 3$ proteoform envelope, where several hemoglobin subunits are also presently detected.

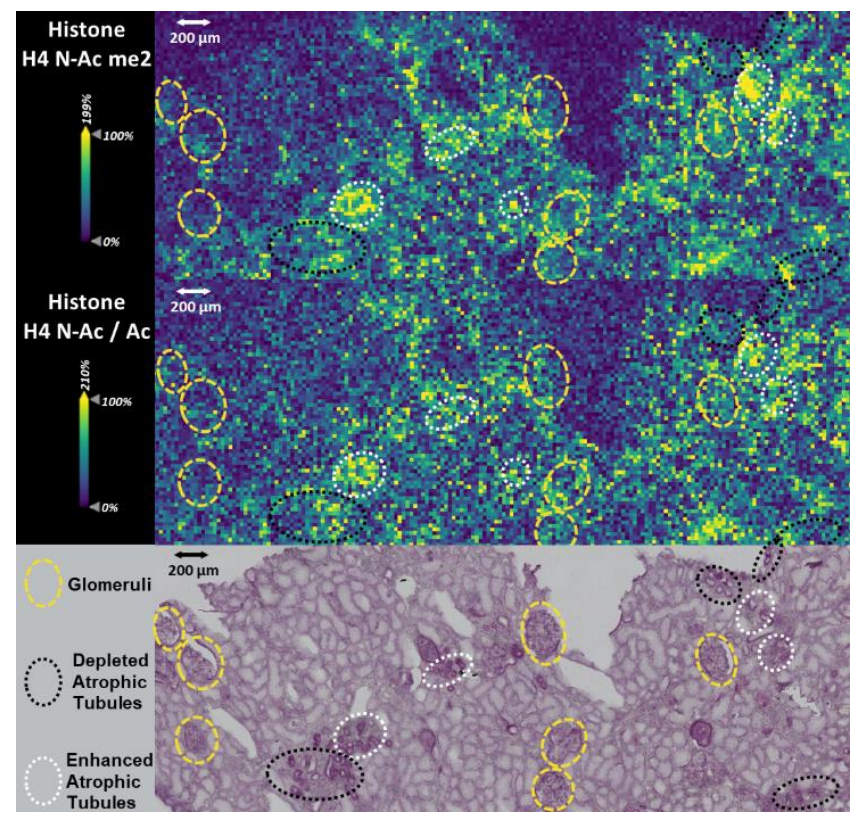

Figure 2. Ion images produced from an expanded view of the MALDI-MSI analysis of a tissue section from a cortical nephrectomy block. The tissue was imaged at $20 \mu \mathrm{m}$ and ion images were obtained by averaging $25 \mathrm{ppm}$ windows for an acetylated and dimethylated histone $\mathrm{H} 4$ proteoform at $\mathrm{m} / \mathrm{z}, 11306.3939$ (-1.12 ppm) and a diacetylated histone $\mathrm{H} 4$ proteoform at $\mathrm{m} / \mathrm{z} 11320.3661$ ($1.74 \mathrm{ppm}$ ). Images are all scaled to $200 \mu \mathrm{m}$ each with separate relative ion abundance. The corresponding microscopic image from the PAS staining post MALDI-MSI is shown at the bottom to highlight atrophic tubular regions with no significant enhancement in signal intensity (black), and areas of tubular atrophy with enhancement of signal (white). Glomeruli are highlighted in yellow. 


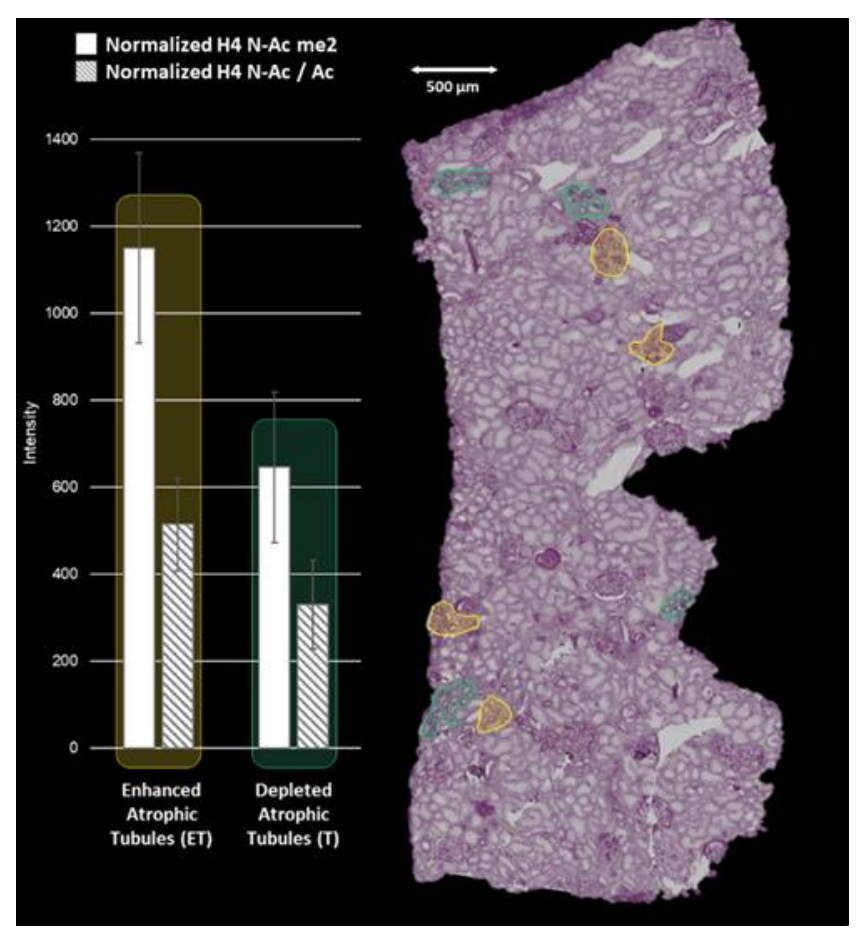

Figure 3. Microscopy image of the PAS-stained section of the healthy nephrectomy post MALDI-MSI. Highlighted within the tissue section are tubular regions identified from MALDI-MSI with differential levels of histone proteoforms, showing higher abundance (ET/yellow) and lower abundance (T/teal) for an acetylated and dimethylated histone H4 proteoform at $\mathrm{m} / \mathrm{z} .11306 .3939$ ($1.12 \mathrm{ppm})$ and a diacetylated histone $\mathrm{H} 4$ proteoform at $\mathrm{m} / \mathrm{z} 11320.3661(-1.74 \mathrm{ppm})$. The bar chart shows extracted mean intensities normalized to the area $\left(\mathrm{mm}^{2}\right)$, with the calculated ratio for each region average below. Error bars represent one standard deviation. 


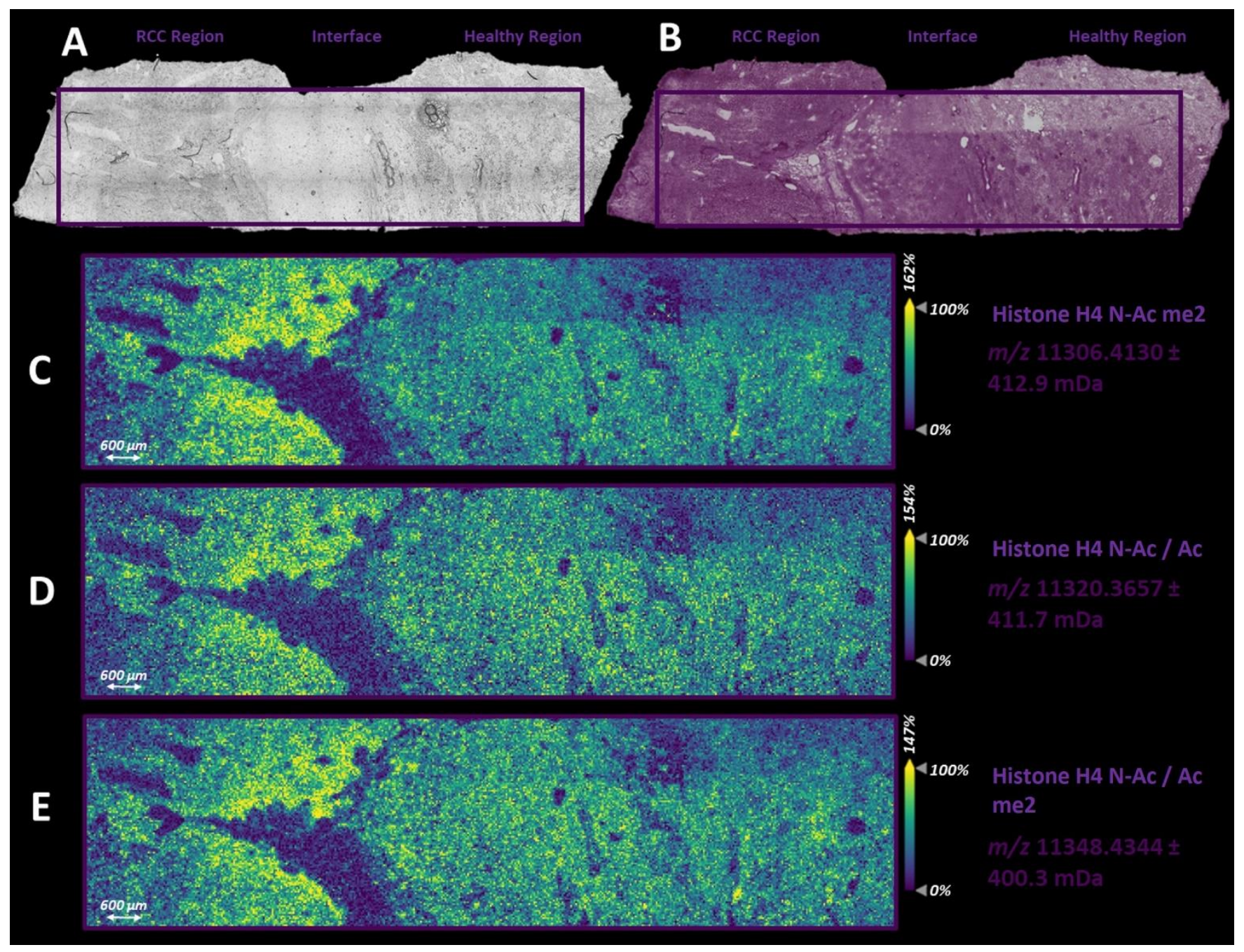

Figure 4. (A) The bright microscopy image of the RCC section prior to MALDI-MSI analysis. (B) The PAS-stained section post MALDI-MSI. Ion images are produced from singular isotopic peak distributions and show an expanded view of the MALDI-MSI analysis at $30 \mu \mathrm{m}$ spatial resolution. Spatial distribution of (C) acetylated and dimethylated histone $\mathrm{H} 4$ proteoform at $\mathrm{m} / \mathrm{z}$ 11306.4130 (0.57 ppm); (D) diacetylated histone H4 proteoform at $\mathrm{m} / \mathrm{z} 11320.3657$ (0.42 ppm); and (E) diacetylated and dimethylated histone $\mathrm{H} 4$ proteoform at $\mathrm{m} / \mathrm{z} 11348.4344(1.78 \mathrm{ppm})$. Scale bars within the ion images are $600 \mu \mathrm{m}$, and each image has a separate ion intensity scale. 